\title{
Expression of three essential antioxidants of Helicobacter pylori in clinical isolates
}

\author{
Yan-yan SHI, Mo CHEN, Yue-xia ZHANG, Jing ZHANG, Shi-gang DING ${ }^{\dagger \neq}$ \\ (Department of Gastroenterology, Peking University Third Hospital, Beijing 100191, China) \\ †E-mail: dingshigang222@163.com \\ Received June 23, 2013; Revision accepted Nov. 11, 2013; Crosschecked Apr. 24, 2014
}

\begin{abstract}
Objective: Helicobacter pylori maintains long-term persistence in the host and combats oxidative stress via many antioxidant proteins, which are expected to be relevant to bacterial-associated gastric diseases. We aimed to investigate the expression of three essential antioxidants in $H$. pylori strains isolated from patients with different clinical outcomes. Methods: Forty $H$. pylori strains were isolated from endoscopic biopsy specimens of gastric mucosa from 13 patients with gastric cancer, 13 with peptic ulcer, and 14 with gastritis. The expression of thioredoxin 1 (Trx1), arginase $(R o c F)$, and alkyl hydroperoxide reductase $(A h p C)$ in $H$. pylori was measured by real-time PCR. Comparisons among multiple sample sets were analyzed using a one-way ANOVA test. Pearson's correlation test was used to assess relationships among multiple continuous variables. Results: Trx1 expression of $H$. pylori in gastric cancer and peptic ulcer tissues was higher than that in tissues with gastritis. RocF expression of $H$. pylori in gastric cancer tissues was higher than that in tissues exhibiting peptic ulcer and gastritis. However, we did not find any differences in $A h p C$ expression in samples from patients with different clinical outcomes. The expression of $\operatorname{Tr} 1$ and RocF had a positive, linear correlation. The expression of $\operatorname{Tr} 1$ and $A h p C$ had a positive correlation without a linear trend. We found no correlation between the expression of RocF and $A h p C$. Conclusions: Our observations indicate that the expression of Trx1 and RocF in H. pylori might be related to gastric carcinogenesis. In H. pylori, the expression of members of the antioxidant system may be correlated and relevant to gastric cancer.
\end{abstract}

Key words: Antioxidant, Gastric cancer, Helicobacter pylori, Oxidative stress

doi:10.1631/jzus.B1300171 Document code: A CLC number: R735.2

\section{Introduction}

Helicobacter pylori is a microaerophilic bacterium and a gastric pathogen that colonizes about $50 \%$ of the world's population. H. pylori infection causes chronic inflammation and significantly increases the risk of developing duodenal and gastric ulcer diseases and gastric cancer. Chronic H. pylori infection is now considered to be the strongest risk factor for gastric cancer (Wroblewski et al., 2010). Recent reports have suggested that exacerbated DNA damage of gastric cells results from inflammation-related oxidative

\footnotetext{
$\$$ Corresponding author

* Project supported by the National Natural Science Foundation of China (No. 81270475)

(C) Zhejiang University and Springer-Verlag Berlin Heidelberg 2014
}

stress induced by reactive oxygen species (ROS) in vivo (Coussens and Werb, 2002).

During the process of colonizing and infiltrating the host cells by $H$. pylori, innate and adaptive inflammatory responses are activated. The activated neutrophils of the host can produce ROS and reactive nitrogen species (RNS), and it has been reported that H. pylori-induced ROS and RNS production in gastric epithelial cells might affect signal transduction and damage DNA in gastric cells, resulting in gastric carcinogenesis (Handa et al., 2010). The inflammatory host response can not only lead to gastric cancer, but also threaten the survival of $H$. pylori. The suppressing effect of oxidative stress species has also been reported in vitro (Qu et al., 2011). Nevertheless, H. pylori successfully survives these conditions and persistently colonizes the gastric mucosa. Therefore, 
mechanisms for detoxification of redox compounds and repair of damaged cell components in H. pylori are particularly important in understanding $H$. pylori pathogenesis and persistence (Wang et al., 2006).

The antioxidant systems in H. pylori appear to be essential for its survival in the host. Studies have indicated that these systems have many components. Thioredoxin-1 (Trx 1$)$ is one of the antioxidants of $H$. pylori (Comtois et al., 2003). H. pylori $\operatorname{Trx} 1$ is similar to a eukaryotic peroxidase reduction system. Trx 1 contains a redox-active site, Cys-Gly-Pro-Cys, and serves as a ubiquitous enzyme that catalyzes the reduction of disulfide bonds (Alamuri and Maier, 2006). Using the purified H. pylori Trx1 and alkyl hydroperoxide reductase (AhpC), Baker et al. (2001) found that $\operatorname{Trx} 1$ can transfer electrons to AhpC. AhpC is an enzyme that reduces peroxides and can protect bacterial and human cells against reactive nitrogen intermediates (Chen et al., 1998). H. pylori Trx1 has also been reported as an arginase (RocF) chaperone. Trx 1 and arginase equip $H$. pylori with a "redox guardian" to overcome oxidative and nitrosative stress (McGee et al., 2006). The functions and relationships of these antioxidants have been reported. However, there is little direct evidence showing the expression of these antioxidants in clinical isolates and their associations with clinical gastric diseases.

In this study, we investigated $\operatorname{Tr} x 1, \operatorname{RocF}$, and AhpC expression in H. pylori strains isolated from tissues exhibiting gastritis, peptic ulcer, and gastric cancer. The relationships among the expression of these antioxidants in $H$. pylori were also assessed using Pearson's correlation test.

\section{Materials and methods}

\subsection{Patient samples}

Gastric biopsies were obtained from a total of 61 patients with gastric cancer, peptic ulcer, and gastritis, who had undergone gastroscopic examination at Peking University Third Hospital (Beijing, China) from 2010 to 2012. All collected tissues had positive results from a rapid urease test, which were confirmed by Warthin-Starry staining. Written informed consent with a signature was obtained from all patients. All tissues were assessed by hematoxylin-eosin (HE) staining. Diagnoses of all the samples were confirmed histologically by two independent pathologists.

\subsection{Culturing of bacterial strains}

Two antral biopsies used for H. pylori culture were collected in tubes containing brucella broth. After homogenizing immediately, the samples were plated on 5\% (v/v) sheep blood agar plates containing amphotericin B $4 \mu \mathrm{g} / \mathrm{ml}$, trimethoprim $4 \mu \mathrm{g} / \mathrm{ml}$, and vancomycin $4 \mu \mathrm{g} / \mathrm{ml}$ (Life Tech, Carlsbad, CA, USA). The plates were incubated for 5 to $7 \mathrm{~d}$ at $37^{\circ} \mathrm{C}$ under microaerophilic conditions using a microaerobic pack (Mitsubishi Gas Chemical Co., Inc., Japan). H. pylori cultures were examined using urease tests and Gram staining. Oxidase tests and catalase tests were used to ensure that the cultures were not contaminated. Bacterial culture was successful in samples from 40 out of 61 infected patients. The 40 samples were isolated from 14 tissues displaying gastritis (including 9 superficial gastritis tissues and 5 atrophic gastritis tissues), 13 peptic ulcer tissues (including 8 gastric ulcer tissues and 5 duodenal ulcer tissues), and 13 gastric cancer tissues (including 2 tissues at an early stage and 11 tissues at an advanced stage; 6 intestinal type tissues, 4 diffuse type tissues, and 3 mixed type tissues).

\subsection{RNA isolation}

RNA extraction from $H$. pylori was performed as described previously (Shi et al., 2013). Briefly, $H$. pylori strains were resuscitated on blood agar plates and harvested to precooled sterile phosphate buffered saline (PBS). After washing with PBS twice, total RNA was isolated from $H$. pylori strains using TRIzol (Invitrogen) following the manufacturer's instructions. RNAs were treated with RNase-free DNase I (Fermentas). The quality of RNA was determined by agarose gel electrophoresis and the quantity by spectrophotometry at $260 \mathrm{~nm}$. RNA was stored at $-80^{\circ} \mathrm{C}$ until needed.

\subsection{Reverse transcription and real-time poly- merase chain reaction $(\mathrm{PCR})$}

Reverse transcription and real-time PCR procedures were performed as described previously (Shi et al., 2013; Zhang et al., 2013). Total RNA (2 $\mu \mathrm{g})$ was reverse-transcribed using the RevertAid First Strand cDNA Synthesis Kit (Fermentas). Real-time PCR was performed using SYBR Green on a Lightcycler 480II 
Real-Time PCR Detection System (Roche, Indianapolis, IN, USA). All results were normalized to $16 \mathrm{~S}$ rRNA amplification. Each reaction was carried out in technical triplicates. Primers were as follows: H. pylori Trx 1: 5'-GGGGTTGCGTTAGTGGATTTTTG-3' (forward primer) and 5'-GACGACTTCGCCATCTTTT GTGA-3' (reverse primer); H. pylori RocF: 5'-TTT ACCTTAGCCTGGATTTAGACA-3' (forward primer) and 5'-GTTGTATTCGGTTACTTCAAGTGC-3' (reverse primer); H. pylori AhpC: 5'-CCCTACAG AAATCATTGCGTTTG-3' (forward primer) and 5'-GGAAAGACACTTGACCAATACCG-3' (reverse primer); 16S rRNA: 5'-CCGCCTACGCGCTCTTT AC-3' (forward primer) and 5'-CTAACGAATAA GCACCGGCTAAC-3' (reverse primer). The relative expression of the target genes was calculated based on cycle threshold $\left(C_{\mathrm{T}}\right)$ measurements. The $\Delta C_{\mathrm{T}}$ values of each sample were calculated as $C_{\mathrm{T}, \text { target gene }}-C_{\mathrm{T}, 16 \mathrm{~S} \text { rRNA }}$ (Shen et al., 2010).

\subsection{Statistical analysis}

A one-way analysis of variance (ANOVA) test followed by a Student-Newman-Keuls (SNK) test was used to analyze the significant differences among multiple sample sets. Pearson's correlation test was used to assess the relationships between multiple continuous variables. Data were presented as mean \pm standard deviation (SD) of three independent experiments. All statistical analyses were performed using SPSS 13.0 software (SPSS Inc., Chicago, IL, USA). $P$-values less than 0.05 were considered statistically significant. GraphPad Prism 5 was used to create the artwork.

\section{Results}

3.1 Expression of $\operatorname{Trx} 1, \mathrm{RocF}$, and $\mathrm{AhpC}$ in $\mathrm{H}$. pylori strains isolated from tissues displaying gastric cancer, peptic ulcer, and gastritis

H. pylori was isolated from 14 gastritis samples, 13 peptic ulcer samples, and 13 gastric cancer samples, and the expression of $\operatorname{Tr} x \mathrm{l}, \operatorname{RocF}$, and $A h p C$ was evaluated. Trxl and $A h p C$ expression was successfully measured in all 40 strains. For the expression of $R o c F, 33$ strains out of 40 were successfully evaluated. The mRNA expression level of Trxl in H. pylori isolated from the gastric cancer and peptic ulcer tis- sues was higher $(P<0.05)$ than that in gastritis tissues (Fig. 1a). No significant difference in $\operatorname{Tr} x 1$ expression was found between the gastric cancer and peptic ulcer samples. The mRNA expression level of RocF in $H$. pylori isolated from the gastric cancer samples was significantly higher $(P<0.05)$ than the level observed for tissues exhibiting gastritis and peptic ulcer (Fig. 1b). However, no significant difference in $R o c F$ expression was found between the gastritis and peptic ulcer samples. The mRNA expression levels of $A h p C$ in $H$. pylori isolated from the gastritis, peptic ulcer, and gastric cancer samples showed no significant differences (Fig. 1c).

\subsection{Correlation among the expression of $R o c F$, Trxl, and $A h p C$ in $H$. pylori strains}

The relationships between $\operatorname{Tr} x 1, \operatorname{RocF}$, and AhpC expression in $H$. pylori strains were assessed using Pearson's correlation test. Trx 1 expression had a positive, linear correlation with $R o c F$ expression $(r=0.411, P<0.05$; Fig. 2a). $\operatorname{Tr} x 1$ expression had a low positive correlation with $A h p C$ expression $(r=0.178)$, but did not present a linear trend $(P>0.05$; Fig. $2 b)$, However, there was no significant correlation $(r=0.009 ; P>0.05)$ between the expression of $R o c F$ and $A h p C$ (Fig. 2c).

\section{Discussion}

It is well known that $H$. pylori infection induces progressive inflammatory changes in the gastric mucosa and increases the risk of peptic ulcer disease and gastric cancer. The antioxidant systems in $H$. pylori were expected to be relevant to bacterialassociated, and especially inflammation-associated, infectious diseases (Wang et al., 2006). In a previous study, we investigated the comparative proteome of clinical H. pylori strains by two-dimensional gel electrophoresis. We reported differential proteins of H. pylori strains isolated from gastritis and gastric cancer, including antioxidants (Zhang et al., 2011). In this study, we investigated the expression of three essential antioxidants of $H$. pylori in clinical isolates to show the relationship between the expression of these antioxidants and different gastric diseases. Correlations between the expression of these antioxidants were also assessed. 

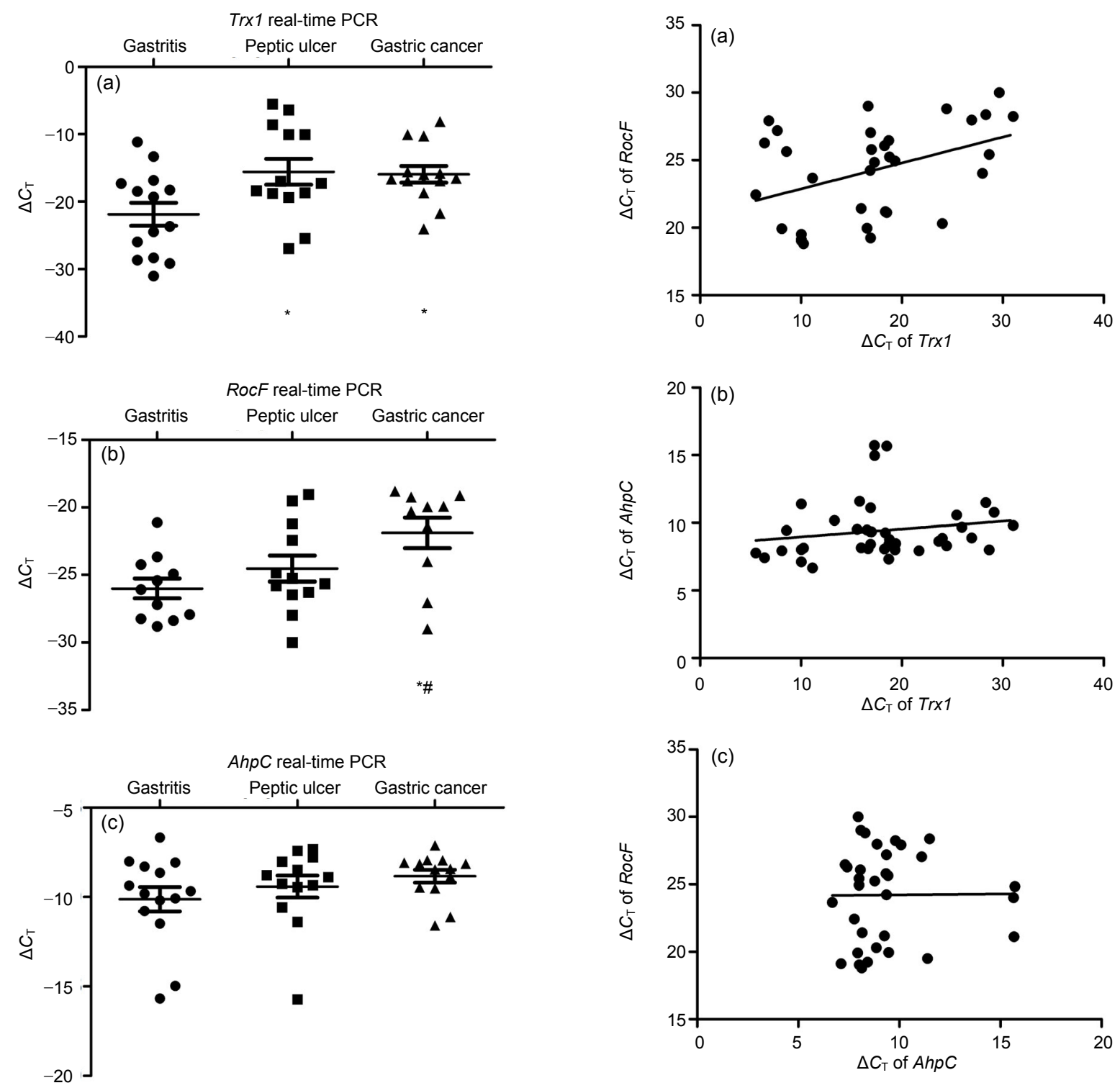

Fig. $1 \operatorname{Tr} x 1$ (a), $\operatorname{RocF}(\mathrm{b})$, and $A h p C$ (c) expression of $H$. pylori in gastric diseases (gastritis, peptic ulcer, and gastric cancer) evaluated by real-time PCR

The mRNA was extracted from $H$. pylori strains isolated from 14 gastritis samples, 13 peptic ulcer samples, and 13 gastric cancer samples. The $\operatorname{Tr} x 1$ (a), $\operatorname{RocF}$ (b), and $\operatorname{Ahp} C$ (c) expression of $H$. pylori was evaluated in these tissues by real-time PCR and normalized to the control gene $16 \mathrm{~S}$ rRNA. The $\Delta C_{\mathrm{T}}$ value of each sample was calculated as $C_{\mathrm{T}, \text { target gene }}-C_{\mathrm{T}, 16 \mathrm{~S} \text { rRNA. }}$. We successfully detected $\operatorname{Tr} x \mathrm{l}$ and $A h p C$ in all 40 strains and $R o c F$ in 33 strains out of 40. ${ }^{*}$ When compared with tissues displaying gastritis, $P<0.05$ was considered statistically significant. ${ }^{\#}$ When compared with peptic ulcer samples, $P<0.05$ was considered statistically significant

Fig. 2 Correlation analysis of $\operatorname{Tr} x 1, \mathrm{RocF}$, and $A$ hpC expression in clinical $\boldsymbol{H}$. pylori isolates

(a) The expression of Trxl in H. pylori strains had a positive, linear correlation with the expression of RocF; (b) The expression of $\operatorname{Tr} x 1$ in $H$. pylori strains had a weakly positive correlation with the expression of $A h p C$, but there was no linear trend; (c) The expression of AhpC in H. pylori strains was not significantly correlated with the expression of $R o c F$

Our results showed that $\operatorname{Trx} 1$ expression of $H$. pylori in clinical gastric cancer and peptic ulcer tissues was higher than that in gastritis tissues. RocF expression of $H$. pylori in gastric cancer tissues was 
higher than that in tissues exhibiting peptic ulcer and gastritis. Furthermore, Pearson's correlation test showed that $\operatorname{Tr} x 1$ and $R o c F$ expression had a positive, linear correlation. Trx 1 is a key protein in many crucial cellular functions, including oxidative stress management (Ritz and Beckwith, 2001). Studies have shown that the expression of intracellular H. pylori $\operatorname{Trx} 1$ is altered in response to a variety of applied extracellular stresses, suggesting that Trx 1 behaves as a stress response element in H. pylori (Windle et al., 2000). Moreover, Trx 1 of $H$. pylori is considered to be an arginase chaperone because of its active site dithiol/disulfide (Berndt et al., 2008). The RocF protein arginase hydrolyzes arginine to ornithine and urea, and urease hydrolyzes urea to carbon dioxide and ammonium, which can neutralize acid. Several reports have shown that the enzymatic pathway of arginase used by $H$. pylori for the production of ornithine and urea can also serve as a mechanism responsible for the lack of protective effect of the immune response and the chronicity of $H$. pylori infection by inhibiting $\mathrm{T}$ cell proliferation and by downregulating eukaryotic NO production (Gobert et al., 2001; Zabaleta et al., 2004). There is a critical disulphide bond for the stability of arginase, which is modulated at the post-translational level by Trx1. Trx1 can convert denatured or suboptimally folded arginase into an optimal three-dimensional catalytically active structure (McGee et al., 2006). In this study, we investigated the relationship between Trx 1 and $R o c F$ expression in clinical isolates of $H$. pylori, based on the supposition that the expression of Trx 1 and $R o c F$ might be correlated and might relate to gastric carcinogenesis.

In this study, we isolated 40 strains from gastric tissues. It has been reported that $H$. pylori RocF in clinical isolates has genetic microheterogeneity and phenotypic variation (Hovey et al., 2007). To avoid genetic variation in $R o c F$ affecting our PCR performance, we designed real-time PCR primers for $R o c F$ in its conserved region suggested by gene sequencing (Hovey et al., 2007). For the expression of RocF, only 33 strains out of 40 were successfully evaluated, probably because of the generally low expression of $R o c F$ shown by the PCR results in this work.

In addition to $R o c F$, we investigated the expression of $A h p C$, one of the most abundant proteins expressed in $H$. pylori and an antioxidant enzyme whose function is essential for reducing different peroxides (Baker et al., 2001). Huang and Chiou (2011) reported the upregulation of $H$. pylori AhpC after treatment with hydrogen peroxide, which suggested that $\mathrm{AhpC}$ might play a part in protecting organisms from damage by ROS. AhpC mutants are hypersensitive to oxygen and organic peroxides and are restricted to atmospheres of less than $2 \%$ oxygen for growth (Olczak et al., 2002). AhpC might assume an important role in combating exogenous peroxides arising from lifelong chronic inflammation (Croxen et al., 2007). Further study using 2-dimensional gel electrophoresis (2-DE) showed that AhpC was expressed in higher amounts in H. pylori strains isolated from three patients with gastric cancer than in strains from three patients with gastritis (Huang et al., 2011). In this study, we assessed the expression of $A h p C$ using real-time PCR in 40 clinical strains from patients, including 13 displaying gastric cancer, 13 displaying peptic ulcer, and 14 displaying gastritis. However, our data did not show any differences in AhpC expression in H. pylori isolated from gastric cancer, peptic ulcer, and gastritis tissues. Although it has been reported that purified Trx1 can transfer electrons to AhpC (Baker et al., 2001), we did not find any relationship between the expression of $A h p C$ and $\operatorname{Tr} x l$ in clinical $H$. pylori isolates. Some previous studies indicated that AhpC was essential for both in vitro growth and gastric colonization (Chalker et al., 2001). However, Croxen et al. (2007) showed that high levels of $\mathrm{AhpC}$ were not required for in vitro growth or for primary gastric colonization. The functions of $\mathrm{AhpC}$ remain unclear and are worthy of further study.

In conclusion, our findings demonstrate that the expression of H. pylori Trx 1 and RocF is higher in gastric cancer tissues than in gastritis tissues. H. pylori infection can induce inflammatory responses followed by ROS/RNS and DNA damage in tissues. Nevertheless, H. pylori successfully survives these conditions by means of antioxidant enzymes, chronically infects gastric epithelium, and induces severe gastric outcomes. This study lends support to the supposition that the antioxidant enzymes in $H$. pylori may be related to the development of gastric cancer from H. pylori infection. Trx 1 and RocF of H. pylori may prove to be prognostic for monitoring varied clinical manifestations of gastrointestinal patients 
infected with $H$. pylori. Further studies should performed to confirm the relationship between $H$. pylori Trx1 and RocF, and to gain more insight into the functions and mechanisms of action of H. pylori Trx 1 and RocF on gastric mucosa, using cell experiments and animal models. Research related to clinical patients and diseases should facilitate a better understanding of the involvement of $H$. pylori antioxidants in pathogenic mechanisms.

\section{Acknowledgements}

We wish to thank Feng-min LU of Peking University (China) for providing technological help.

\section{Compliance with ethics guidelines}

Yan-yan SHI, Mo CHEN, Yue-xia ZHANG, Jing ZHANG, and Shi-gang DING declare that they have no conflict of interest.

All procedures followed were in accordance with the ethical standards of the responsible committee on human experimentation (institutional and national) and with the Helsinki Declaration of 1975, as revised in 2008 (5). Informed consent was obtained from all patients for being included in the study.

\section{References}

Alamuri, P., Maier, R.J., 2006. Methionine sulfoxide reductase in Helicobacter pylori: interaction with methionine-rich proteins and stress-induced expression. J. Bacteriol., 188(16):5839-5850. [doi:10.1128/JB.00430-06]

Baker, L.M., Raudonikiene, A., Hoffman, P.S., et al., 2001. Essential thioredoxin-dependent peroxiredoxin system from Helicobacter pylori: genetic and kinetic characterization. J. Bacteriol., 183(6):1961-1973. [doi:10.1128/ JB.183.6.1961-1973.2001]

Berndt, C., Lillig, C.H., Holmgren, A., 2008. Thioredoxins and glutaredoxins as facilitators of protein folding. Biochim. Biophys. Acta, 1783(4):641-650. [doi:10.1016/j.bbamcr. 2008.02.003]

Chalker, A.F., Minehart, H.W., Hughes, N.J., et al., 2001. Systematic identification of selective essential genes in Helicobacter pylori by genome prioritization and allelic replacement mutagenesis. J. Bacteriol., 183(4):12591268. [doi:10.1128/JB.183.4.1259-1268.2001]

Chen, L., Xie, Q.W., Nathan, C., 1998. Alkyl hydroperoxide reductase subunit $\mathrm{C}(\mathrm{AhpC})$ protects bacterial and human cells against reactive nitrogen intermediates. Mol. Cell, 1(6):795-805. [doi:10.1016/S1097-2765(00)80079-9]

Comtois, S.L., Gidley, M.D., Kelly, D.J., 2003. Role of the thioredoxin system and the thiol-peroxidases Tpx and Bcp in mediating resistance to oxidative and nitrosative stress in Helicobacter pylori. Microbiology, 149(1):121-129. [doi:10.1099/mic.0.25896-0]
Coussens, L.M., Werb, Z., 2002. Inflammation and cancer. Nature, 420(6917):860-867. [doi:10.1038/nature01322]

Croxen, M.A., Ernst, P.B., Hoffman, P.S., 2007. Antisense RNA modulation of alkyl hydroperoxide reductase levels in Helicobacter pylori correlates with organic peroxide toxicity but not infectivity. J. Bacteriol., 189(9):33593368. [doi:10.1128/JB.00012-07]

Gobert, A.P., McGee, D.J., Akhtar, M., et al., 2001. Helicobacter pylori arginase inhibits nitric oxide production by eukaryotic cells: a strategy for bacterial survival. PNAS, 98(24):13844-13849. [doi:10.1073/pnas.241443798]

Handa, O., Naito, Y., Yoshikawa, T., 2010. Helicobacter pylori: a ROS-inducing bacterial species in the stomach. Inflamm. Res., 59(12):997-1003. [doi:10.1007/s00011010-0245-x]

Hovey, J.G., Watson, E.L., Langford, M.L., et al., 2007. Genetic microheterogeneity and phenotypic variation of Helicobacter pylori arginase in clinical isolates. BMC Microbiol., 7(1):26. [doi:10.1186/1471-2180-7-26]

Huang, C.H., Chuang, M.H., Lo, W.L., et al., 2011. Alkylhydroperoxide reductase of Helicobacter pylori as a biomarker for gastric patients with different pathological manifestations. Biochimie, 93(7):1115-1123. [doi:10. 1016/j.biochi.2011.03.008]

McGee, D.J., Kumar, S., Viator, R.J., et al., 2006. Helicobacter pylori thioredoxin is an arginase chaperone and guardian against oxidative and nitrosative stresses. J. Biol. Chem., 281(6):3290-3296. [doi:10.1074/jbc.M506139200]

Olczak, A.A., Olson, J.W., Maier, R.J., 2002. Oxidative-stress resistance mutants of Helicobacter pylori. J. Bacteriol., 184(12):3186-3193. [doi:10.1128/JB.184.12.3186-3193. 2002]

Qu, W., Zhou, Y., Sun, Y., et al., 2011. Identification of $S$-nitrosylation of proteins of Helicobacter pylori in response to nitric oxide stress. J. Microbiol., 49(2):251-256. [doi:10.1007/s12275-011-0262-7]

Ritz, D., Beckwith, J., 2001. Roles of thiol-redox pathways in bacteria. Ann. Rev. Microbiol., 55(1):21-48. [doi:10. 1146/annurev.micro.55.1.21]

Shen, R., Pan, S., Qi, S., et al., 2010. Epigenetic repression of microRNA-129-2 leads to overexpression of SOX4 in gastric cancer. Biochem. Biophys. Res. Commun., 394(4): 1047-1052. [doi:10.1016/j.bbrc.2010.03.121]

Shi, Y., Liu, L., Zhang, T., et al., 2013. The involvement of Helicobacter pylori thioredoxin-1 in gastric carcinogenesis. J. Med. Microbiol., 62(Pt 8):1226-1234. [doi:10. 1099/jmm.0.056903-0]

Wang, G., Alamuri, P., Maier, R.J., 2006. The diverse antioxidant systems of Helicobacter pylori. Mol. Microbiol., 61(4):847-860. [doi:10.1111/j.1365-2958.2006.05302.x]

Windle, H.J., Fox, A., NíEidhin, D., et al., 2000. The thioredoxin system of Helicobacter pylori. J. Biol. Chem., 275(7):5081-5089. [doi:10.1074/jbc.275.7.5081]

Wroblewski, L.E., Peek, R.M., Wilson, K.T., 2010. Helicobacter pylori and gastric cancer: factors that modulate 
disease risk. Clin. Microbiol. Rev., 23(4):713-739. [doi:10.1128/CMR.00011-10]

Zabaleta, J., McGee, D.J., Zea, A.H., et al., 2004. Helicobacter pylori arginase inhibits $\mathrm{T}$ cell proliferation and reduces

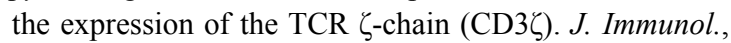
173(1):586-593. [doi:10.4049/jimmunol.173.1.586]

Zhang, H.H., Cai, A.Z., Wei, X.M., et al., 2013. Characterization of cancer stem-like cells in the side population cells of human gastric cancer cell line MKN-45. $J$. Zhejiang Univ.-Sci. B (Biomed. \& Biotechnol.), 14(3): 216-223. [doi:10.1631/jzus.B1200102]

Zhang, Y.N., Ding, S.G., Huang, L.H., et al., 2011. Comparative proteome analysis of Helicobacter pylori clinical strains by two-dimensional gel electrophoresis. $J$. Zhejiang Univ. Sci. B (Biomed. \& Biotechnol.), 12(10): 820-827. [doi:10.1631/jzus.B1000445]

\section{中文概要:}

\section{本文题目：幽门螺杆菌临床分离菌株中三个重要抗氧化物的表达水平及相关性分析}

Expression of three essential antioxidants of Helicobacter pylori in clinical isolates

研究目的：幽门螺杆菌 (Helicobacter pylori) 长期在宿主体内定植, 并通过多种抗氧化蛋白对抗氧化 应激, 目前的研究认为该过程与其所致的胃粘膜疾病相关。我们拟临床研究不同胃粘膜疾 病来源的 H. pylori 菌株中三个关键抗氧化物的表达。

创新要点: 首次分析 H. pylori 临床分离菌株中氧化应激相关因子与胃粘膜疾病类型的关系, 并对三种 因子的表达水平进行相关性分析。突出临床病人感染 H. pylori 中氧化应激相关分子的表达 特点, 对研究 H. pylori 各氧化应激相关分子与临床胃粘膜疾病的关系, 以及临床病人感染 的 H. pylori 中各个氧化应激相关分子之间的关系具有重要的意义。

研究方法: 在胃镜检查时, 取患者胃粘膜组织进行 H. pylori 培养, 共分离培养出 40 例临床菌株, 其 中 13 例来自胃癌患者, 13 例来自消化性溃疡患者, 14 例来自胃炎患者。用荧光实时定量 聚合酶链式反应（PCR）的方法测定 H. pylori 临床分离菌株中硫氧还蛋白-1（Trx 1）、精 氨酸酶 $(R o c F)$ 以及烷基过氧化物酶 $(A h p C)$ 的表达水平。多组样本间比较用单因素方 差分析法, 并用 Pearson 相关性分析法评价多组连续变量的相关性。

重要结论: H. pylori Trxl mRNA 表达水平在胃癌和消化性溃疡分离菌株中显著高于胃炎分离株, $H$. pylori RocF mRNA 表达水平在胃癌患者分离菌株中显著高于胃炎和消化性溃疡分离株, 我们未发现胃炎、消化性溃疡和胃癌患者分离的 H. pylori 菌株中 $A h p C$ 的表达差异。H. pylori RocF 的表达水平与 $\operatorname{Tr} x 1$ 呈显著线性正相关, $A h p C$ 的 mRNA 表达水平与 $\operatorname{Tr} x 1$ 呈非 线性正相关, $R o c F$ 与 $A h p C$ 的 mRNA 表达水平无相关性。结果表明, H. pylori Trx l 和 RocF 的表达水平可能与胃癌相关, 在 H. pylori 菌株中, 抗氧化系统的各个因子可能相互联系, 在胃癌发病中发挥作用。

关键词组: 抗氧化物; 胃癌; 幽门螺杆菌; 氧化应激 